\title{
What the WSAVA can do for you
}

As a member of the BSAVA you are
also a member of the World Small
Animal Veterinary Association
(WSAVA). Many of its initiatives and the
wealth of resources it creates are highly
relevant to veterinary professionals in
the UK. Read on for the WSAVA
benefits available to you.

Taking a stand on global issues

The WSAVA takes the lead in raising awareness of global animal welfare issues and was busy during 2017 focusing on the following.

\begin{abstract}
Condemning the dog and cat meat trade In September the WSAVA expressed its opposition to the dog and cat meat trade in a position statement calling for the rigorous enforcement of existing laws. It also called for new controls and regulations where current legislation does not exist, aimed at banning what it regards as an inhumane and dangerous practice. The position statement is available at http://bit.ly/2Cw1ehU.
\end{abstract}

\section{Highlighting the plight of brachycephalic animals}

During FECAVA-WSAVA World Congress, the WSAVA co-hosted an expert panel session on issues raised by brachycephalic breeding, following a lecture stream dedicated to hereditary disease and

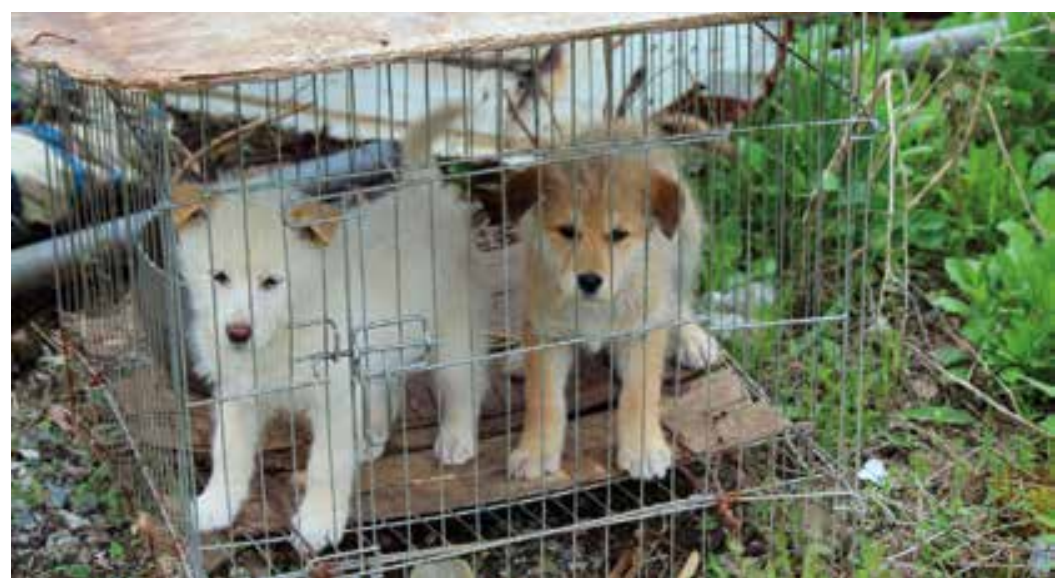

The WSAVA is opposed to the dog and cat meat trade and is actively working towards banning this practice.

Courtesy of Change For Animals Foundation. the importance of responsible breeding. The panel urged the 200 + attendees to 'dare to speak out' on this important issue and provided recommendations to help veterinary professionals to work with pet owners to reduce the suffering of affected animals. Details of the recommendations are available at http://bit.ly/2yb4cFp with follow up activities planned for this year.

\section{Working to raise standards of animal welfare globally}

Members of the WSAVA's Animal Wellness and Welfare Committee continued the development of the latest in its 'Standardization Guidelines' series. Its Animal Welfare Guidelines will set out recommendations for the veterinary team, following every interaction with a patient to ensure that, in addition to providing the very best health advice and therapy, they offer evidence-based information and guidance to ensure the health, welfare and safety of the animal, the owner and themselves. The Guidelines will be launched during the 2018 World Congress in Singapore from 25-28 September.

\section{Driving best practice in companion animal medicine}

Educating the profession globally is one of the WSAVA's most important priorities. During 2017 it delivered continuing professional development (CPD) lectures in more than 30 countries, spanning four continents. It focuses its efforts in parts of the world in which companion animal practice is still developing and gives members in these countries unique access to global experts. The BSAVA is a long-term supporter of the WSAVA's CPD efforts in sub-Saharan Africa.

In September 2017, the WSAVA launched its Global Dental Guidelines to support veterinary professionals to provide a higher standard of dental care to patients. Members of the WSAVA Dental Guidelines Committee hope that the new guidelines will help to bridge a significant gap in veterinary education globally and encourage a greater emphasis on dentistry in the veterinary curriculum. You can download the Guidelines at http://www.wsava.org/guidelines/ global-dental-guidelines.

Co-chairs of the WSAVA Global Dental Guidelines Committee, Dr Brook Niemiec and Dr Jerzy Gawor, will present a day-long seminar to introduce the Guidelines during BSAVA Congress 2018

\section{Building a global veterinary community}

The WSAVA is working to create a collaborative and supportive community of global veterinary peers through initiatives including: 
The WSAVA-Hill's Next Generation Award to recognize the work of a veterinary professional graduated within the past ten years, who has contributed significantly to the betterment of companion animals, the veterinary profession and society at large

- The WSAVA Global One Health Award to recognize an individual or organization which has promoted an aspect of One Health relevant to companion animals

- The WSAVA Award for Scientific Achievement to recognize an individual who has made a significant contribution to the field of small animal medicine

- The WSAVA Award for Meritorious Service to recognize a veterinary professional who has contributed meritorious service to the veterinary profession.

\section{'Getting them to Congress'}

The WSAVA runs a scheme through which its financially stronger member associations support member representatives from developing countries to attend WSAVA World Congress and benefit from the learning opportunities on offer.

\section{Support for Mission Rabies}

The WSAVA supports Mission Rabies, a charity led by Dr Luke Gamble - the recipient of the 2016 WSAVA One Health Award. His team's efforts have resulted in more than 700,000 dogs and cats being vaccinated; 90,000 sterilised and more than 1.6 million children educated on rabies prevention in Asia and Africa. The WSAVA's charitable Foundation is also focused on developing small animal veterinary associations, infrastructure and sustainability in sub-Saharan Africa.

\section{What's new for 2018?}

The WSAVA has a range of new initiatives underway for 2018 including:

- A new website (wwww.wsava.org), optimized for access on all internet-enabled devices, has been launched. It is an online hub for the latest veterinary resources, knowledge and news from the WSAVA itself and from its member associations

- It has launched a Professional Wellness and Wellbeing Committee in order to help safeguard the mental health and well-being of veterinary professionals globally

- It has launched a new Veterinary Therapeutics Guidelines Group to focus on building equality of therapeutic access proper use and minimum veterinary pharmacy requirements around the world.

Make sure you get the best out of your BSAVA membership by visiting www.wsava.org for more information.

\section{The WSAVA at a glance}

The WSAVA represents more than 200,000 veterinary professionals worldwide through 105 member associations and is focused on enhancing the clinical care of companion animals. One of its core activities is the development of WSAVA Global Standardization Guidelines in key areas of veterinary practice. These Guidelines aim to support members by setting standards for care and recommending best practice.

The WSAVA's global veterinary community is already bringing great benefit to members of our profession but the WSAVA has ambitions to make it even stronger and to provide a global voice for veterinary professionals. The association welcomes more active involvement from members in the UK so, if you would like to make 2018 the year that you contribute to your profession at a global level, please contact WSAVA Chief Executive Officer Arpita Bhose at yourwsava@wsava.org for more information.

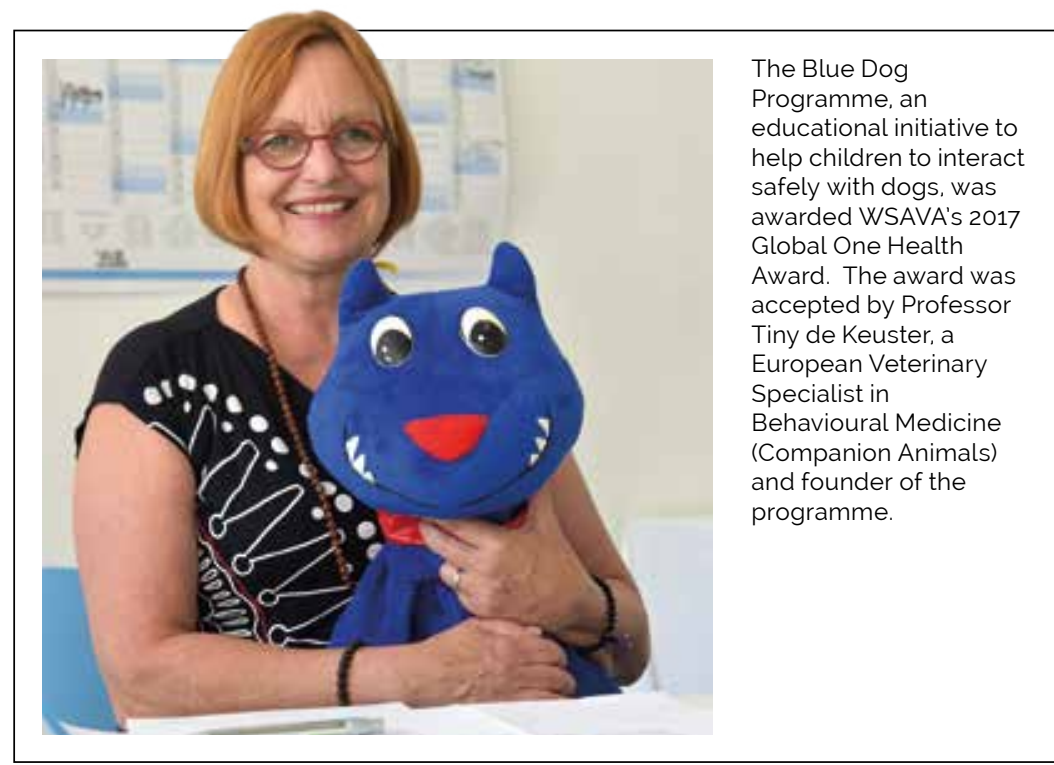

